\title{
Maternal low-protein diet during mouse pre-implantation development induces vascular dysfunction and altered renin-angiotensin-system homeostasis in the offspring
}

\author{
Adam J. Watkins ${ }^{1}$, Emma S. Lucas ${ }^{1}$, Christopher Torrens ${ }^{2}$, Jane K. Cleal ${ }^{2}$, Lauren Green ${ }^{1}$, Clive Osmond ${ }^{3}$, \\ Judith J. Eckert ${ }^{1,2}$, William P. Gray ${ }^{4}$, Mark A. Hanson ${ }^{2}$ and Tom P. Fleming ${ }^{1}$ \\ ${ }^{1}$ School of Biological Sciences, University of Southampton, Bassett Crescent East, Southampton SO16 7PX, UK \\ ${ }^{2}$ Institute of Developmental Sciences, Developmental Origins of Health and Disease (DOHaD) Division, School of Medicine, \\ University of Southampton, Southampton General Hospital, Southampton SO16 6YD, UK \\ ${ }^{3}$ MRC Epidemiology Resource Centre, Southampton General Hospital, Southampton SO16 6YD, UK \\ ${ }^{4}$ Division of Clinical Neurosciences, School of Medicine, University of Southampton, Southampton General Hospital, Southampton \\ SO16 6YD, UK \\ (Received 15 July 2009 - Revised 8 December 2009 - Accepted 10 December 2009 - First published online 4 February 2010)
}

\begin{abstract}
Environmental perturbations during early mammalian development can affect aspects of offspring growth and cardiovascular health. We have demonstrated previously that maternal gestational dietary protein restriction in mice significantly elevated adult offspring systolic blood pressure. Therefore, the present study investigates the key mechanisms of blood pressure regulation in these mice. Following mating, female MF-1 mice were assigned to either a normal-protein diet (NPD; $18 \%$ casein) or an isocaloric low-protein diet throughout gestation (LPD; $9 \%$ casein), or fed the LPD exclusively during the pre-implantation period ( $3 \cdot 5 \mathrm{~d})$ before returning to the NPD for the remainder of gestation (Emb-LPD). All offspring received standard chow. At 22 weeks, isolated mesenteric arteries from LPD and Emb-LPD males displayed significantly attenuated vasodilatation to isoprenaline $(P=0.04$ and $P=0.025$, respectively), when compared with NPD arteries. At 28 weeks, stereological analysis of glomerular number in female left kidneys revealed no significant difference between the groups. Real-time RT-PCR analysis of type 1a angiotensin II receptor, $\mathrm{Na}^{+} / \mathrm{K}^{+}$ATPase transporter subunits and glucocorticoid receptor expression in male and female left kidneys revealed no significant differences between the groups. LPD females displayed elevated serum angiotensin-converting enzyme (ACE) activity $(P=0 \cdot 044)$, whilst Emb-LPD males had elevated lung ACE activity $(P=0.001)$, when compared with NPD offspring. These data demonstrate that elevated offspring systolic blood pressure following maternal gestational protein undernutrition is associated with impaired arterial vasodilatation in male offspring, elevated serum and lung ACE activity in female and male offspring, respectively, but kidney glomerular number in females and kidney gene expression in male and female offspring appear unaffected.
\end{abstract}

CVD: Maternal diet: Pre-implantation embryo development

The development of adult CVD has been associated typically with classical risk factors such as poor diet, a sedentary lifestyle, smoking and genetic predisposition. However, the 'Developmental Origins of Health and Disease' (DOHaD) hypothesis identifies the uterine environment and associated maternal nutritional status as critical components in the origin of postnatal cardiovascular and metabolic disease ${ }^{(1-3)}$. The DOHaD concept proposes that maternal nutritional status influences embryonic and fetal growth and physiology so that the setting of homeostatic mechanisms matches the nutrient availability in postnatal life. However, mismatch between pre- and postnatal nutrient environments is associated with adult onset disease ${ }^{(1-3)}$.
Rodent and domestic animal studies have demonstrated that maternal gestational undernutrition can alter cardiovascular homeostasis in the offspring ${ }^{(4-8)}$. The precise mechanisms underlying dietary programmed hypertension are likely to be multifactorial and complex, originating from more than one individual pathway. Vascular dysfunction in adult programmed animals in response to prolonged gestational undernutrition is characterised by an enhanced constrictive and impaired endothelial-dependent dilatation phenotype. In the sheep, maternal nutrient restriction encompassing either preconception or early gestation enhances vasoconstriction and attenuates vasodilatation in offspring coronary, thoracic and renal $\operatorname{arteries}^{(9-11)}$. However, a more prolonged maternal gestational

Abbreviations: ACE, angiotensin-converting enzyme; Emb-LPD, low-protein diet for the first $3 \cdot 5 \mathrm{~d}$ before switching to normal diet for the remainder of gestation; LPD, low-protein diet; NPD, normal-protein diet; pEC50, - log effective concentration equal to $50 \%$ of the maximal response; RAS, renin-angiotensin system; SBP, systolic blood pressure.

* Corresponding author: Dr Adam Watkins, fax +442380 594459, email ajw7@soton.ac.uk 
nutrition restriction associates with a higher offspring blood pressure and altered baroreflex responses to noradrenaline infusion $^{(12)}$. In the rat, male offspring of dams either globally undernourished (30\% less food than controls), or fed a low-protein diet (LPD), throughout pregnancy display significantly attenuated vascular responsiveness to both endothelial-independent and -dependent vasodilators ${ }^{(4-8,13)}$. However, the magnitude of this vascular impairment was unaffected by a subsequent postnatal high-fat dietary challenge $^{(13)}$, highlighting the detrimental effect of poor maternal nutrition during pregnancy on vascular function.

Along with vascular function, homeostatic regulation of the renin-angiotensin system (RAS) also appears susceptible to programming in response to dietary and environmental challenges during gestation. The production of angiotensin II, a potent vasoconstrictor, from angiotensin $\mathrm{I}$ is mediated by angiotensin-converting enzyme $(\mathrm{ACE})^{(14)}$. The binding of angiotensin II to its receptor ${ }^{(15)}$ results in vasoconstriction, renal tubular $\mathrm{Na}$ reabsorption and aldosterone and vasopressin secretion $^{(16)}$. Angiotensin II has also been implicated in endothelial dysfunction through its ability to increase oxidative stress $^{(17)}$. As a result, altered RAS regulation has been associated with the development of the hypertensive state ${ }^{(15)}$ and with several cardiovascular and renal pathologies ${ }^{(18,19)}$. The role of RAS in the aetiology of programmed adult hypertension following maternal nutrient restriction has been demonstrated via the normalisation of blood pressure in hypertensive adult offspring from LPD-fed rat dams after administration of the ACE inhibitor, captopril ${ }^{(20)}$, or through the blockade of the angiotensin $\mathrm{I}$ receptor $\left(\mathrm{AT}_{1} \mathrm{R}\right)$ with $\operatorname{losartan}^{(21)}$. Offspring from rat dams fed LPD during gestation display significantly altered mRNA and protein levels for the angiotensin I and II receptors $\left(A_{1} R\right.$ and $\left.A T_{2} R\right)$ in kidney tissue ${ }^{(22,23)}$. Maternal LPD has also been shown to alter expression levels of the glucocorticoid receptor and the $\mathrm{Na} / \mathrm{K}$-adenosine triphosphatase subunits in the kidney ${ }^{(24)}$. These findings have led to the suggestion that maternal undernutrition could alter renal $\mathrm{Na}$ retention, contributing to the increased systolic blood pressure (SBP) observed in these animals.

The pre-implantation period of development has been shown to be particularly sensitive to changes in its immediate environment, whether in vivo or in vitro, resulting in a myriad of changes in offspring phenotype ${ }^{(25-29)}$. Altered cardiovascular and metabolic phenotype, including raised SBP and diastolic blood pressure and altered glucose homeostasis, have been identified in human in vitro fertilisation children compared with age-matched controls ${ }^{(30)}$. In the sheep, culture of preimplantation embryos in the presence of serum resulted in significantly altered rates of blastocyst development, and elevated growth of placental and fetal tissues ${ }^{(31)}$. Impaired blastocyst and fetal development have also been reported following the culture of mouse pre-implantation embryos under high ammonia ${ }^{(32)}$ or reduced oxygen ${ }^{(33)}$ environments. Studies from our laboratory have demonstrated that maternal LPD diet targeted exclusively to the pre-implantation period in rodents significantly alters blastocyst cell numbers and gene expression patterns in fetal liver tissue ${ }^{(26,29,34,35)}$, whilst elevating offspring postnatal growth, behavioural patterns and mean lifelong $\mathrm{SBP}^{(29)}$.

Whilst the development of offspring hypertension following maternal gestational undernutrition is well established, the mechanisms and pathways involved are still poorly defined. In the present study we evaluate for the first time key mechanisms of adult blood pressure regulation, in order to gain a better understanding of how maternal undernutrition during discrete windows of gestation can programme disease in the adult offspring.

\section{Methods}

\section{Animal treatments}

All experiments were conducted using protocols approved by the UK Home Office and local ethics committee. Female MF-1 mice were bred in-house (University of Southampton Biomedical Research Facility) and maintained on a 07.00 to 19.00 hours light cycle with controlled temperature and standard chow and water ad libitum. All offspring and archived tissues investigated in the present study were taken from the same cohort of animals as described previously ${ }^{(29)}$. Briefly, virgin MF-1 females (aged 7-8.5 weeks) were mated naturally with MF-1 studs (Harlan Link Ltd, Bicester, Oxon, UK) and randomly assigned to either (a) a normal protein diet (NPD; $18 \%$ casein) throughout gestation, (b) an isocaloric lowprotein diet (LPD; $9 \%$ casein) throughout gestation, or (c) the LPD for the first $3.5 \mathrm{~d}$ before switching to the NPD for the remainder of gestation (Emb-LPD). The morning after mating, females were individually coded and fed such that the experimenter was blinded to the diet each female received, and subsequently did not know what dietary treatment any offspring pertained to. Nineteen litters of each treatment group were generated. Mothers from the day of birth, and offspring (after weaning at 3 weeks), were fed standard chow. Litter size was adjusted to six at birth (three per sex) and the sexes caged separately from weaning. Previously, we demonstrated that these dietary regimens were sufficient to induce mean lifelong hypertension in both male (elevated by $3.2 \mathrm{mmHg}$ ) and female (elevated by $3.0 \mathrm{mmHg}$ ) offspring as assessed by tail-cuff plethysmography ${ }^{(29)}$, and that female Emb-LPD offspring displayed increased body weight from birth for up to 28 weeks (increased by $11 \%$ at birth, and $8.3 \%$ at 28 weeks, respectively, over controls ${ }^{(29)}$. Analysis of the mean lifelong SBP (measurements taken at 9, 15 and 21 weeks of age) of the cohort of males and females used within all experiments within the present study (fifty-five, fifty-seven and sixty-one NPD, Emb-LPD and LPD offspring, respectively) revealed that both Emb-LPD and LPD offspring had significantly elevated mean lifelong SBP when compared with the control offspring used (NPD 103.93 (SEM 0.61) mmHg; Emb-LPD 107.46 (SEM 0.43) $\mathrm{mmHg}$; LPD 107.3 (SEM 0.53) $\mathrm{mmHg}$; $P<0.001)$. Therefore, the mice in the cohort analysed in the present study were considered as being representative of the entire population $^{(29)}$ from which they were selected.

\section{Mesenteric artery vasoreactivity}

Vascular function in male offspring was assessed at 22 weeks in isolated small mesenteric artery segments following cervical dislocation as described previously ${ }^{(7,8,13,36)}$. Male offspring alone were analysed in order to minimise potential variation caused from the differing levels of circulating oestrogen in female offspring during the oestrous cycle, and the 
effect this has on vascular responsiveness ${ }^{(37)}$. Briefly, small mesenteric artery segments (luminal diameter about 100$300 \mu \mathrm{m})$ were isolated and mounted on a wire myograph (Danish Myo Technology A/S, Aarhus, Denmark). Following normalisation, cumulative concentration response curves were measured for the $\alpha_{1}$-adrenergic agonist phenylephrine $\left(10^{-9}\right.$ to $\left.10^{-4} \mathrm{~mol} / \mathrm{l}\right)$. After pre-constriction with a submaximal dose of phenylephrine ( $80 \%$ of maximal excitatory concentration; $\mathrm{EC}_{80}$ ), cumulative concentration response curves to the endothelial-dependent and -independent vasodilators acetylcholine $\left(10^{-9}\right.$ to $\left.10^{-5} \mathrm{~mol} / \mathrm{l}\right)$ and isoprenaline $\left(10^{-10}\right.$ to $\left.10^{-6} \mathrm{~mol} / \mathrm{l}\right)$, respectively, and the NO donor, sodium nitroprusside $\left(10^{-11}\right.$ to $\left.10^{-5} \mathrm{~mol} / \mathrm{l}\right)$, were conducted in that order in the same mesenteric arteries from seven or eight individual males, all from different litters. All drugs were purchased from Sigma (Poole, Dorset, UK).

\section{Kidney glomerular counting}

Due to the fact that male offspring alone had been analysed for the study of mesenteric artery responsiveness (for reasons given above), only female kidneys were analysed in order to maintain a balanced number of male and female offspring for the final analysis of organ allometry at 28 weeks (organ allometry data previously detailed by Watkins et al. ${ }^{(29)}$ ). This also kept the present study in line with our previous study $^{(36)}$, making it possible to compare results. Stereological analysis of kidney glomerular number was conducted as previously described ${ }^{(36)}$. Briefly, left kidneys from female offspring were perfusion-fixed with neutral buffered formalin (BDH Laboratory Supplies, Poole, Dorset, UK). Kidney volume was determined by suspended immersion into neutral buffered formalin placed on a balance, with the increase in weight recorded being equal to the volume of the kidney. Three separate measurements of volume per kidney were taken and an average calculated. Fixed kidneys were then processed into glycol methacrylate (JB-4 Embedding Kit; Polysciences Europe $\mathrm{GmbH}$, Eppelheim, Germany) and exhaustively sectioned at $2 \mu \mathrm{m}$. Every 100th and 110th section was analysed using the Stereo Investigator software (Microbrightfield, Williston, VT, USA). An unbiased counting frame $\left(500 \mu \mathrm{m}^{2}\right)$ was placed randomly over the 100th section; glomeruli present within the 110th but absent from the 100th section were counted and an estimate of kidney glomerular number was obtained using the fractionator principle ${ }^{(38)}$.

\section{RNA extraction and quantitative RT-PCR for gene expression} analysis

Whole left kidneys from eight male and eight female offspring per treatment group at 28 weeks, archived from our previous study ${ }^{(29)}$, were powdered and the RNA extracted using the RNeasy ${ }^{\circledR}$ Lipid Tissue Mini Kit (QIAGEN, Crawley, West Sussex, UK) according to the manufacturer's instructions. On-column DNase I digestion was performed to remove contaminating genomic DNA. RNA was reverse transcribed to cDNA using the ImProm ${ }^{\mathrm{TM}} \mathrm{II}$ kit (Promega, Southampton, Hants, UK), according to the manufacturer's instructions. Intron-spanning primers used in the present study are detailed in Table 1. For real-time PCR, a mastermix was prepared consisting of $10 \mu \mathrm{l} 2 \mathrm{X}$ Precision Mastermix containing

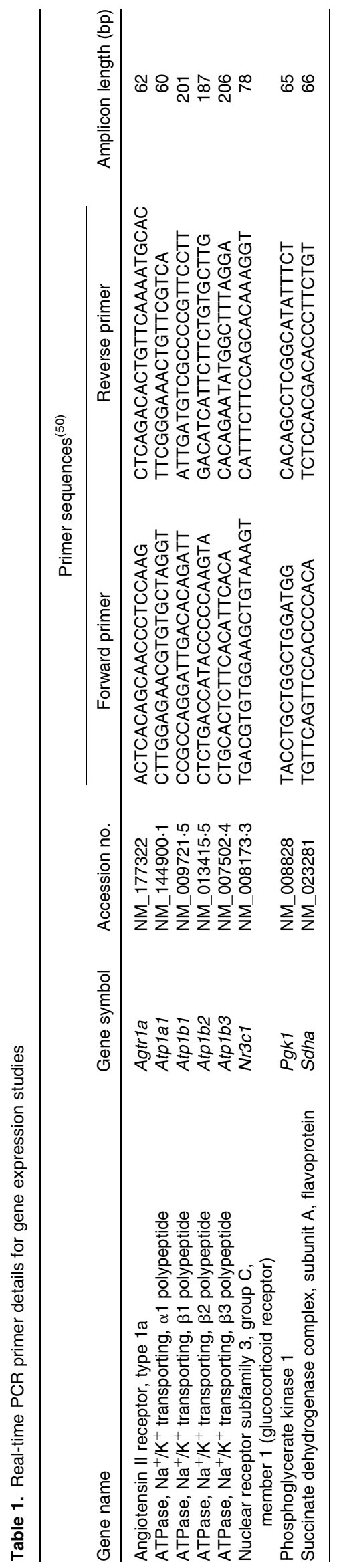


SYBRgreen (PrimerDesign, Southampton, Hants, UK), $1 \cdot 2 \mu 1$ primer mix (containing $5 \mu \mathrm{M}$ each forward and reverse primers) and $7.8 \mu \mathrm{l}$ water per reaction. Mastermix was aliquotted to ninety-six-well PCR plates (Axygen, Union City, CA, USA) in $19 \mu \mathrm{l}$ volumes and $5 \mathrm{ng}$ cDNA was added to sample wells, or water to control (no template) wells, giving a final reaction volume of $20 \mu \mathrm{l}$. All samples were run in triplicate. An inter-plate calibrator was included on all runs to control for inter-assay variation. Amplification and detection were performed using a DNA Engine thermal cycler and Chromo4 Real-Time Detector and data were acquired using Opticon Monitor version 3.1 software (Bio-Rad, Hemel Hempstead, Herts, UK). A post-amplification melting curve confirmed the presence of specific products for each primer set. Data were normalised to the expression of succinyl dehydrogenase $\alpha$ (Sdha) and phosphoglycerate kinase 1 (Pgkl) using geNorm software (Ghent University, Ghent, Belgium $)^{(39)}$, with which we determined these to be the two most stable reference genes in adult mouse kidney (data not shown).

\section{Angiotensin-converting enzyme activity}

Serum and lung ACE activity was analysed as previously described $^{(28,40)}$. Briefly, samples were incubated in the presence of hippuryl-L-histidine-L-leucine acetate salt (Sigma, Poole, Dorset, UK) solution in buffer at $37^{\circ} \mathrm{C}$ followed by the addition of cyanuric chloride (Sigma) in 1,4 dioxane (Sigma). Each sample was analysed in duplicate, with four readings per duplicate analysed at $380 \mathrm{~nm}$. Samples containing only serum or lung lysate and buffer were used as negative controls. Sample readings were analysed against a standard curve ranging from $50 \mu \mathrm{M}$ to $1 \mathrm{mM}$, prepared in buffer and treated exactly as the samples. Serum and lung ACE activity was expressed as nmol hippurate formed per $\mathrm{ml}$ serum per min or per mg protein per min, respectively.

\section{Statistical analysis}

For analysis of vascular responsiveness data, a four-parameter logistic equation using non-linear regression was used to obtain the $-\log$ effective concentration equal to $50 \%$ of the maximal response (pEC50) and maximum response for each of the cumulative concentration response curves analysed. Differences in maximal response and pEC50 were analysed using two-way ANOVA (Prism 3.0, GraphPad Software Inc., San Diego, CA, USA) with Bonferroni post hoc correction as described previously ${ }^{(7,13,36)}$. Kidney glomerular number and gene transcript analysis and ACE data were analysed using a multilevel random effects regression model (SPSS version 15; SPSS, Inc., Chicago, IL, USA) taking into account the hierarchical nature of the data with between-mother and within-mother variation and different parameters measured from individual animals ${ }^{(28,29,41)}$. Therefore, statistical differences identified between treatment groups were independent of maternal origin of litter, gestational litter size and body weight, unless otherwise stated. All data are presented as mean values with their standard errors of the mean.

\section{Results}

\section{Mesenteric artery vasoreactivity}

Isolated male arteries from all treatment groups displayed a concentration-dependent vasoconstriction to the $\alpha_{1}$-adrenergic agonist phenylephrine, which was not different between treatments (Fig. 1(a)). Following pre-constriction with phenylephrine, no difference in responsiveness to the endotheliumdependent vasodilator acetylcholine was observed between treatment groups (Fig. 1(b)). However, Emb-LPD males displayed an attenuated response to the $\beta$-adrenoceptor agonist isoprenaline when compared with NPD arteries (\% maximal response 62.22 (SEM 4.59) v. 76.11 (SEM 3.58), respectively; $P=0.025$ ) whilst the response in LPD arteries was shifted significantly to the right (significantly different $\mathrm{pEC}_{50}$ ) when compared with NPD arteries ( $\mathrm{pEC}_{50}, 6.99$ (SEM 0.07) and 7.56 (SEM 0.13), respectively; $P=0.04$; Fig. 1(c)), both of which were independent of litter size and body weight effects. No differences in response to sodium nitroprusside were observed between the treatment groups.

\section{Kidney glomerular counts}

Stereological analysis of female left kidneys at 28 weeks revealed no difference in mean glomerular number between the treatment groups, or in mean kidney weight or mean kidney volume (Fig. 2).

\section{Kidney gene expression analysis}

Real-time analysis of type 1a angiotensin II receptor (AgtrlA), the $\alpha 1, \beta 1,2$ and 3 subunits of the $\mathrm{Na}^{+} / \mathrm{K}^{+}$ATPase transporter (Atpla1, Atplb1, Atplb2, Atplb3) and glucocorticoid receptor $(\mathrm{Nr} 3 \mathrm{cl}$ ) mRNA expression in male and female left kidneys revealed no significant differences between any of the treatment groups (Table 2).

\section{Serum and lung angiotensin-converting enzyme activity}

Analysis of serum ACE activity showed no differences between male treatment groups; however, LPD females had an elevated activity compared with NPD females (135.45 (SEM 11.65) v. 113.17 (SEM 9.48) nmol hippurate formed $/ \mathrm{ml}$ serum per min, respectively; Fig. 3(a); $P=0.044$ ). Analysis of lung ACE activity showed that Emb-LPD males had an elevated activity when compared with NPD and LPD males (3.98 (SEM 0.48) v. 1.69 (SEM 0.16) and 2.33 (SEM 0.29) nmol hippurate formed/mg protein per min, respectively; Fig. 3(b); $P \leq 0.005)$. Whilst Emb-LPD females had an elevated activity when compared with LPD females (3.31 (SEM 0.55) v. 2.29 (SEM 0.41) nmol hippurate formed/mg protein per min, respectively; Fig. 3(b); $P=0.05)$, there was no difference between Emb-LPD females and LPD and control females. All differences were independent of litter size effects; however, body weight at 28 weeks of age had a negative influence on female lung ACE activity ( $-0.104 \mathrm{nmol}$ hippurate formed $/ \mathrm{mg}$ protein per min per $\mathrm{g}$ increase in body weight; $P=0 \cdot 018$ ). 

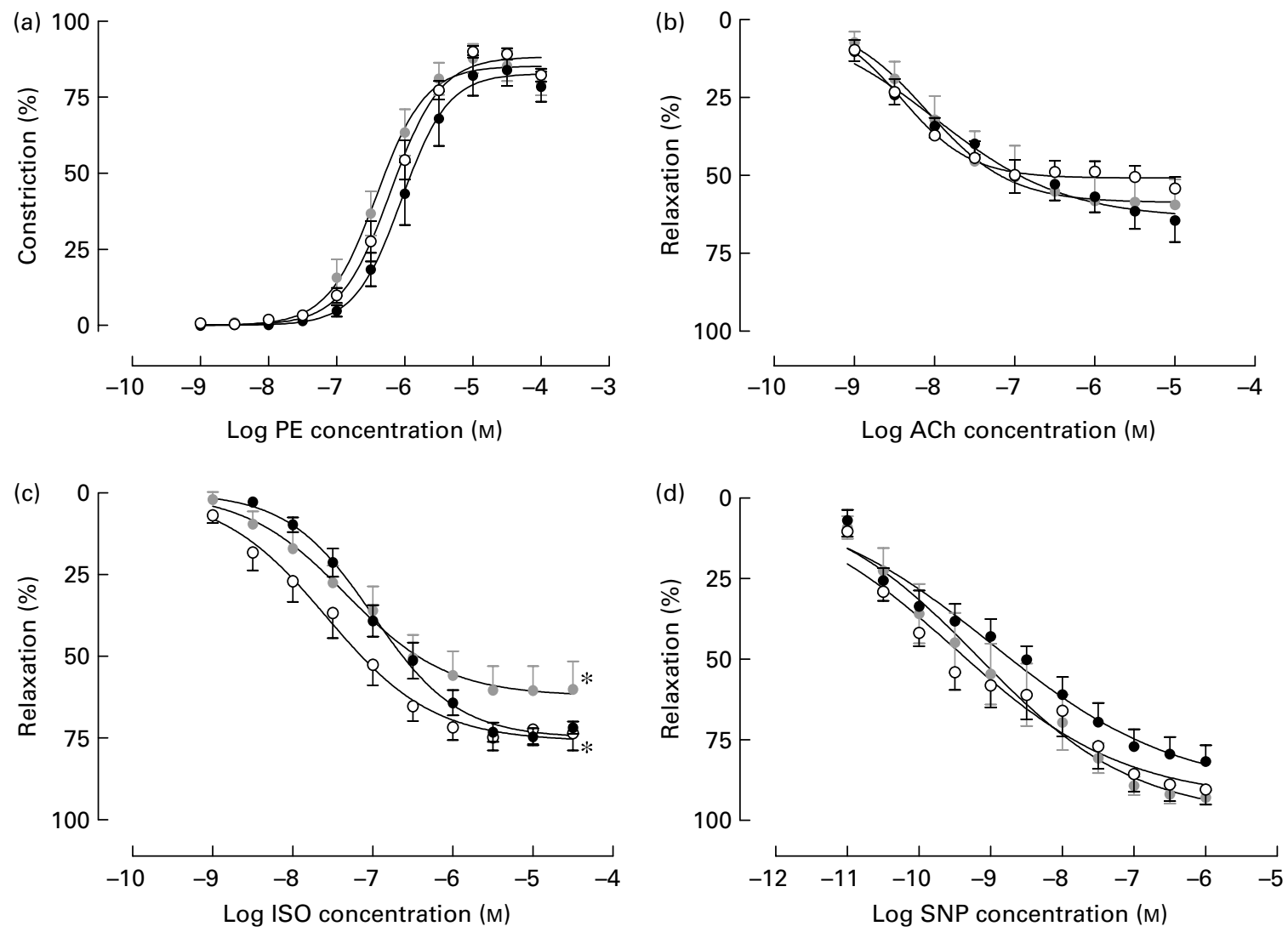

Fig. 1. Vasoreactivity of isolated mesenteric arteries from male offspring at 22 weeks of age from dams fed a low-protein diet throughout gestation (LPD; $\bullet$ ) or exclusively during the pre-implantation period (Emb-LPD; O) compared with arteries of offspring of normal-protein diet (NPD)-fed dams (O). Cumulative additions of (a) phenylephrine (PE) and, after pre-constriction with PE, of the vasodilators (b) acetylcholine (ACh), (c) isoprenaline (ISO) and (d) sodium nitroprusside (SNP). Values are means of seven to eight males, each from different litters representing each treatment group, with standard errors represented by vertical bars. ${ }^{*}$ Response was significantly different from that of the NPD arteries $(P<0.05)$.

\section{Discussion}

The earliest stages of development have been highlighted as critically sensitive periods during which perturbations, either in vivo or in vitro, can have long-term effects on offspring health and physiology $(25-29,31,33,36)$. Previously, we have demonstrated that a maternal LPD treatment exclusively during the pre-implantation period (the first $3.5 \mathrm{~d}$ of gestation;
Emb-LPD) or throughout gestation (LPD) significantly elevated offspring $\mathrm{SBP}^{(29)}$. Therefore, the present study investigated the mechanisms underlying the observed elevated SBP in a cohort of these same offspring, focusing on vascular function, the RAS and changes in nephron number and kidney gene expression patterns. We observed impaired vasodilatation in male LPD and Emb-LPD offspring, elevated patterns
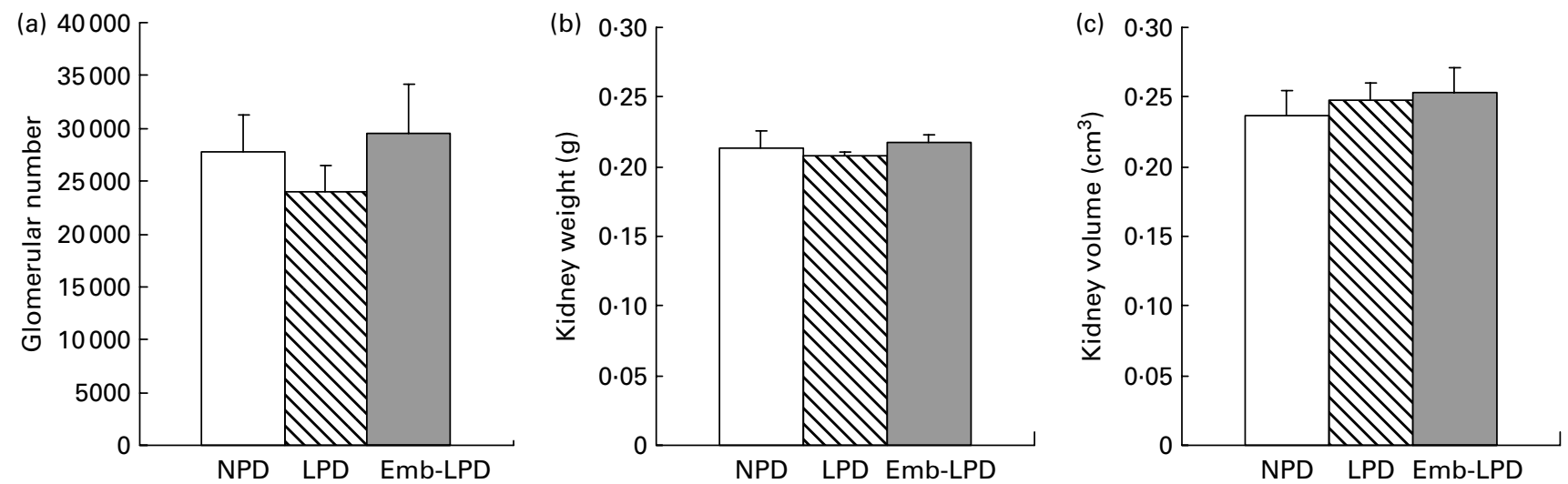

Fig. 2. Effect of maternal low-protein diet either throughout gestation (LPD) or exclusively during the pre-implantation period (Emb-LPD) compared with maternal normal-protein diet (NPD) on kidney glomerular number (a), kidney weight (b) and kidney volume (c) in female offspring at 28 weeks. Values are means for six kidneys per treatment, each from different litters representing each treatment group, with standard errors represented by vertical bars. 
Table 2. Real-time RT-PCR relative expression values from male and female kidney tissue*

(Mean values with their standard errors)

\begin{tabular}{|c|c|c|c|c|c|c|}
\hline \multirow[b]{3}{*}{ Gene of interest† } & \multicolumn{6}{|c|}{ Relative expression values } \\
\hline & \multicolumn{2}{|c|}{ NPD } & \multicolumn{2}{|c|}{ LPD } & \multicolumn{2}{|c|}{ Emb-LPD } \\
\hline & Mean & SEM & Mean & SEM & Mean & SEM \\
\hline \multicolumn{7}{|l|}{ Agtr1a } \\
\hline Males & 1.52 & 0.12 & 1.55 & $0 \cdot 11$ & 1.48 & 0.12 \\
\hline Females & $1 \cdot 28$ & 0.13 & 1.58 & 0.13 & 1.28 & 0.12 \\
\hline \multicolumn{7}{|l|}{$N r 3 c 1$} \\
\hline Males & 1.5 & 0.11 & 1.55 & $0 \cdot 12$ & 1.71 & 0.06 \\
\hline Females & 1.49 & 0.13 & 1.34 & 0.09 & 1.54 & 0.13 \\
\hline \multicolumn{7}{|l|}{ Atp1a1 } \\
\hline Males & $1 \cdot 15$ & 0.11 & 1.23 & $0 \cdot 11$ & 1.2 & 0.06 \\
\hline Females & 1.37 & 0.14 & 1.31 & $0 \cdot 13$ & 1.25 & 0.11 \\
\hline \multicolumn{7}{|l|}{ Atp $1 b 1$} \\
\hline Males & 1.09 & 0.09 & 1.22 & 0.06 & 1.24 & 0.07 \\
\hline Females & 1.23 & 0.13 & 1.22 & 0.13 & 1.22 & 0.09 \\
\hline \multicolumn{7}{|l|}{ Atp1b2 } \\
\hline Males & 1.15 & 0.16 & 1.29 & 0.11 & 1.24 & 0.09 \\
\hline Females & 1.33 & 0.11 & 1.53 & 0.15 & 1.26 & 0.07 \\
\hline \multicolumn{7}{|l|}{ Atp1b3 } \\
\hline Males & 1.37 & 0.12 & 1.37 & 0.1 & 1.37 & 0.07 \\
\hline Females & 1.30 & 0.14 & 1.28 & $0 \cdot 12$ & $1 \cdot 17$ & 0.06 \\
\hline
\end{tabular}

NPD, normal-protein diet; LPD, isocaloric low-protein diet; Emb-LPD, low-protein diet for the first $3.5 \mathrm{~d}$ before switching to normal diet for the remainder of gestation.

*Eight males and eight females, each from different litters representing each treatment group.

†For gene names, see Table 1

of serum and lung ACE activity in LPD females and EmbLPD males, respectively, but no change in kidney glomerular number in females or kidney gene expression in male and female offspring. These data further strengthen the association between the predisposition to chronic adult-onset diseases and an impaired uterine environment during critical stages of gestation and especially before implantation.

Our first major finding was that isolated mesenteric arteries from Emb-LPD and LPD male offspring displayed significantly attenuated responsiveness to the $\beta$-adrenoceptor agonist, isoprenaline. The association of CVD and attenuated vascular responsiveness has been demonstrated previously in offspring from LPD fed rats ${ }^{(4,7,8,42)}$ and in offspring from dietary induced obese mice ${ }^{(43)}$. The present data, however, identify the importance of nutrition during the pre-implantation period in the maintenance of vascular tone. Since endothelial-dependent vasodilatation appeared unaffected, as gauged by similar responses to acetylcholine between treatment groups, it might suggest that the vascular defect lies in altered smooth muscle signalling via cAMP, rather than the bioavailability of responsiveness to NO. Isoprenaline produces vasodilatation through activation of $\mathrm{cAMP}^{(44)}$; however, in mouse mesenteric arteries this is dependent on a functioning endothelial NO synthase ${ }^{(45)}$. One suggestion is that basal endothelial NO release can modulate isoprenaline responses by inhibiting phosphodiesterase breakdown of $\mathrm{cAMP}^{(46)}$. From the present study the role of $\mathrm{NO}$ is unclear, as responses were not performed in the presence of an NO synthase inhibitor such as $N$-nitro-L-arginine-methyl ester. Furthermore, reductions in the expression of guanylate cyclase or intracellular cGMP concentrations have been demonstrated in models of

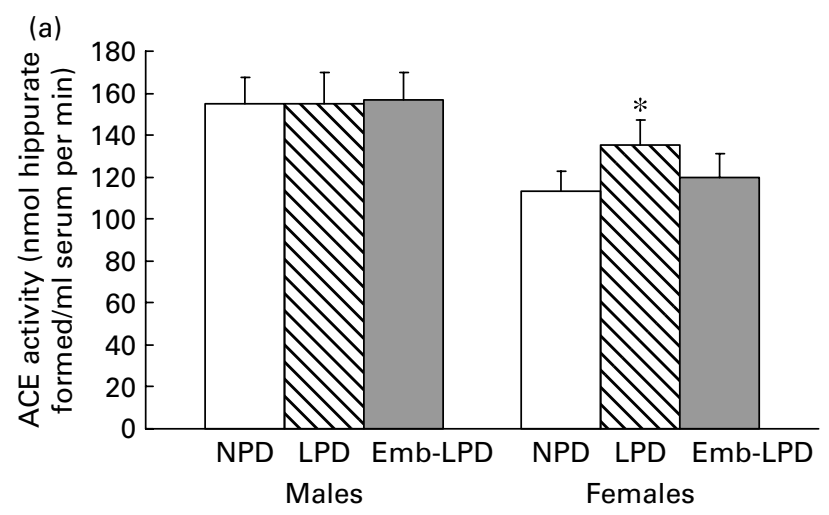

(b)

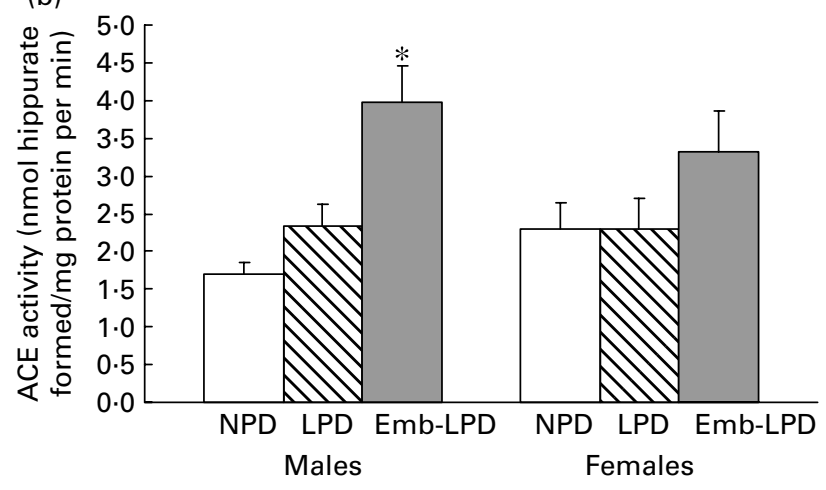

Fig. 3. Enzymic activity of serum (a) and lung tissue (b) angiotensin-converting enzyme (ACE) activity in male and female offspring at 28 weeks. NPD, normalprotein diet; LPD, low-protein diet; Emb-LPD, low-protein diet for the first 3.5d before switching to normal diet for the remainder of gestation. Values are means for seventeen to nineteen males and females per treatment, each from different litters representing each treatment group, with standard errors represented by vertical bars. ${ }^{*}$ Mean value was significantly different from that of the NPD group $(P<0.05)$.

impaired endothelium-independent responsiveness ${ }^{(47-49)}$. It is therefore possible that alterations in guanylate cyclase or cGMP may be contributing to the blunted vasodilatation responses observed in the male offspring within the present study; however, this would require further investigation to confirm. Similarly, whether differences in vasodilatation exist for female offspring from LPD and Emb-LPD mothers also remains to be determined. However, vascular responsiveness at different stages of the oestrous cycle would have to be determined so that both dietary and hormonal effects could be quantified independently.

The second major alteration in postnatal phenotype that we observed was that female LPD offspring displayed significantly elevated serum ACE activity, whilst Emb-LPD males displayed elevated lung ACE activity. The association between $\mathrm{ACE}$ and SBP is well documented. $\mathrm{ACE}^{-1-}$ mice are hypotensive, with a mean SBP about $30 \mathrm{mmHg}$ lower than wild-type mice ${ }^{(50,51)}$. Hypertensive offspring from LPDfed rat dams show a normalisation in their SBP after administration of the ACE inhibitor captopril ${ }^{(20)}$ whilst hypertensive mice developing from embryos genetically programmed to develop at a slower rate, or from in vitro cultured embryos, display significantly elevated serum ACE activities ${ }^{(28,40)}$. In human subjects, serum ACE levels are significantly higher in hypertensive as opposed to normotensive subjects ${ }^{(52)}$, with 
serum ACE levels correlating positively with plasma angiotensin II levels ${ }^{(53)}$. The programming of elevated serum and tissue ACE activity has also been demonstrated following maternal cortisol infusion ${ }^{(54)}$, and co-occurring with adult elevated SBP in response to pre-implantation embryo culture conditions ${ }^{(28)}$ and maternal $\operatorname{diet}^{(36)}$. As in our previous studies $^{(28,40)}$ we observed sex- and tissue-specific responses in ACE activity across treatment groups. In vivo, serum $\mathrm{ACE}$ is derived from the cleavage of endothelial-bound somatic ACE from the lungs, vasculature and kidneys. As we only assessed the activity of ACE in the serum and lungs, we are not able to comment on the activity of other somatic sources of ACE, their relative contribution to the overall activity in the serum or in possible differences between the sexes.

The third finding was that female left kidney glomerular number was unaltered in response to maternal dietary protein levels. Significant reductions in both kidney size and glomerular number have been reported in response to maternal LPD in the rat ${ }^{(55)}$, mouse ${ }^{(56)}$ and with decreased birth weight in humans ${ }^{(57)}$. However, several studies have demonstrated programmed elevated blood pressure in the absence of significant changes in glomerular number ${ }^{(58-60)}$. Whilst reductions in glomerular number may reflect compromised kidney development during gestation in females, in response to restricted maternal nutrition, nephrogenesis in the mouse and rat is not complete until several days after birth ${ }^{(61)}$. As all dietary challenges ceased by time of birth any reduction in glomerular number occurring during gestation in response to the LPD could potentially be compensated for during early postnatal life. This may be one reason for the similar glomerular numbers, kidney weights and volumes observed in the present study in the female offspring. In the present study, only female offspring were analysed and so it would also be of interest to determine whether glomerular number was similarly unaffected in males.

Examination of gene expression in the left kidneys of male and female offspring found no differences in the expression of glucocorticoid receptor transcripts or in any of the $\mathrm{Na}^{+} / \mathrm{K}^{+}$ ATPase subunits examined. This is in contrast to studies in the rat model, whereby exposure to maternal LPD has led to reported 2-fold increases in glucocorticoid receptor mRNA expression, as well as in transcripts for the $\alpha 1$ and $\beta 1$ subunits of the $\mathrm{Na}^{+} / \mathrm{K}^{+}$ATPase ${ }^{(24)}$, in association with raised SBP in the order of $20-30 \mathrm{mmHg}$. The present study also found no differences in expression of the angiotensin II type $1 \mathrm{~A}$ receptor (Agtrla) between the kidneys of treated and control offspring. In the rat model, while up-regulated Agtrla mRNA has been observed in LPD offspring from one study at 4 weeks of age ${ }^{(23)}$, another study reported no difference in expression of this transcript ${ }^{(22)}$. This may reflect differences in the timing and severity of the dietary manipulation between these two studies, whereby the altered Agtrla expression was observed after exposure of dams to a $6 \%$ casein diet from day 12 of pregnancy ${ }^{(23)}$ but not after exposure to a $9 \%$ diet from the day of mating until delivery ${ }^{(22)}$. This discrepancy reinforces the hypothesis that the specific mechanistic origins of the programmed hypertension phenotype are driven by the timing and severity of the nutritional challenge.

It is of interest to compare the relative difference in postnatal phenotype observed in the present study with those occurring in response to maternal LPD during oocyte maturation $^{(21)}$. During the present study, we observed endothelialindependent impairments in vasodilatation, elevated ACE activity profiles, but no changes in glomerular number. However, in response to the LPD during oocyte maturation, we observed impaired endothelial-dependent and -independent vasodilatation, no changes in ACE activity and an elevation in glomerular number ${ }^{(36)}$. As previously discussed in detail $^{(36)}$, these changes may reflect the different times during the reproductive cycle during which the LPD was administered (gamete maturation $v$. post-fertilisation development). Alternatively, they may reflect the contribution of the paternal genome, absent during oocyte maturation ${ }^{(62,63)}$. A third distinction is the connection between early postnatal phenotype and adult physiology and cardiovascular health present in this and previous associated studies ${ }^{(29)}$ which appear absent in the offspring derived from the LPD administered during oocyte maturation ${ }^{(36)}$. Whilst both dietary protocols result in hypertensive offspring, the mechanisms underlying this state appear both similar (vascular responsiveness) and distinct (ACE activity, body weight and kidney morphology).

In conclusion, we have demonstrated that maternal LPD given either exclusively during pre-implantation embryo development, or throughout gestation, results in an impaired arterial vasodilatory capacity in male offspring, and altered activity of serum and lung ACE activity in female and male offspring, respectively. However, kidney glomerular number in females, and expression of key RAS component transcripts and blood volume regulators in both male and female offspring were unaffected. These findings demonstrate that maternal undernutrition encompassing the earliest stages of development has an impact upon interacting vascular, signalling and enzymic pathways which associate with the development of an elevated offspring SBP phenotype ${ }^{(29)}$. These changes in offspring homeostatic regulation may have occurred in response to adaptations made during embryonic and/or fetal development to maximise postnatal development in an anticipated nutritional environment, but which then predispose the offspring to adult disease. Collectively, the present results offer novel insights into the mechanisms behind the association between poor pre-implantation maternal diet and adult cardiovascular dysfunction.

\section{Acknowledgements}

The present study was supported by the Biotechnology and Biological Sciences Research Council (BBSRC; grant no. BBF007450), the National Institutes of Health, USA as part of the National Institute of Child Health and Human Development (NICHD) National Cooperative Program on Female Health and Egg Quality under cooperative agreement (grant no. U01 HD04435) and the Gerald Kerkut Charitable Trust. M. A. H. is supported by the British Heart Foundation. We thank the Biomedical Facility, University of Southampton, for their technical support. We also acknowledge the help of Stephanie Marfy-Smith with the preparation of the kidney tissue for gene expression analyses.

A. J. W., E. S. L., C. T., J. K. C., W. P. G., J. J. E. and T. P. F. designed the research; A. J. W., E. S. L. and L. G. performed the research; A. J. W., E. S. L., C. T. and C. O. 
analysed the data; A. J. W., E. S. L., C. T., M. A. H. and T. P. F. wrote the manuscript.

There are no conflicts of interest associated with the present study.

\section{References}

1. Barker DJ (2002) Fetal programming of coronary heart disease. Trends Endocrinol Metab 13, 364-368.

2. Hanson MA \& Gluckman PD (2008) Developmental origins of health and disease: new insights. Basic Clin Pharmacol Toxicol 102, 90-93.

3. McMillen IC \& Robinson JS (2005) Developmental origins of the metabolic syndrome: prediction, plasticity, and programming. Physiol Rev 85, 571-633.

4. Brawley L, Itoh S, Torrens C, et al. (2003) Dietary protein restriction in pregnancy induces hypertension and vascular defects in rat male offspring. Pediatr Res 54, 83-90.

5. Edwards LJ \& McMillen IC (2002) Periconceptional nutrition programs development of the cardiovascular system in the fetal sheep. Am J Physiol Regul Integr Comp Physiol 283, R669-R679.

6. Langley SC \& Jackson AA (1994) Increased systolic blood pressure in adult rats induced by fetal exposure to maternal low protein diets. Clin Sci (Lond) 86, 217-222.

7. Torrens C, Brawley L, Barker A, et al. (2003) Maternal protein restriction in the rat impairs resistance artery but not conduit artery function in pregnant offspring. $J$ Physiol 547, 77-84.

8. Torrens C, Brawley L, Anthony F, et al. (2006) Folate supplementation during pregnancy improves offspring cardiovascular dysfunction induced by protein restriction. Hypertension 47, 982-987.

9. Cleal JK, Poore KR, Boullin JP, et al. (2007) Mismatched pre- and postnatal nutrition leads to cardiovascular dysfunction and altered renal function in adulthood. Proc Natl Acad Sci U S A 104, 9529-9533.

10. Khan OA, Torrens C, Noakes DE, et al. (2005) Effects of pre-natal and early post-natal undernutrition on adult internal thoracic artery function. Eur J Cardiothorac Surg 28, 811-815.

11. Torrens C, Snelling TH, Chau R, et al. (2009) Effects of pre- and periconceptional undernutrition on arterial function in adult female sheep are vascular bed dependent. Exp Physiol 94, $1024-1033$.

12. Gopalakrishnan GS, Gardner DS, Rhind SM, et al. (2004) Programming of adult cardiovascular function after early maternal undernutrition in sheep. Am J Physiol Regul Integr Comp Physiol 287, R12-R20.

13. Torrens C, Hanson MA, Gluckman PD, et al. (2009) Maternal undernutrition leads to endothelial dysfunction in adult male rat offspring independent of postnatal diet. $\mathrm{Br} J$ Nutr 101, $27-33$.

14. Skeggs LT, Kahn JR \& Shumway NP (1956) The preparation and function of the hypertensin-converting enzyme. $J$ Exp Med 103, 295-299.

15. Higuchi S, Ohtsu H, Suzuki H, et al. (2007) Angiotensin II signal transduction through the $\mathrm{AT}_{1}$ receptor: novel insights into mechanisms and pathophysiology. Clin Sci (Lond) $\mathbf{1 1 2}$ 417-428.

16. Unger T, Chung O, Csikos T, et al. (1996) Angiotensin receptors. J Hypertens Suppl 14, S95-S103.

17. Mehta PK \& Griendling KK (2007) Angiotensin II cell signaling: physiological and pathological effects in the cardiovascular system. Am J Physiol Cell Physiol 292, C82-C97.

18. Lucius R, Gallinat S, Busche S, et al. (1999) Beyond blood pressure: new roles for angiotensin II. Cell Mol Life Sci 56, $1008-1019$.
19. Unger $\mathrm{T}$ (2002) The role of the renin-angiotensin system in the development of cardiovascular disease. Am J Cardiol 89, 3A-9A.

20. Langley-Evans SC \& Jackson AA (1995) Captopril normalises systolic blood pressure in rats with hypertension induced by fetal exposure to maternal low protein diets. Comp Biochem Physiol A Physiol 110, 223-228.

21. Sherman RC \& Langley-Evans SC (2000) Antihypertensive treatment in early postnatal life modulates prenatal dietary influences upon blood pressure in the rat. Clin Sci (Lond) 98, 269-275.

22. McMullen S, Gardner DS \& Langley-Evans SC (2004) Prenatal programming of angiotensin II type 2 receptor expression in the rat. Br J Nutr 91, 133-140.

23. Vehaskari VM, Stewart T, Lafont D, et al. (2004) Kidney angiotensin and angiotensin receptor expression in prenatally programmed hypertension. Am J Physiol Renal Physiol 287, F262-F267.

24. Bertram C, Trowern AR, Copin N, et al. (2001) The maternal diet during pregnancy programs altered expression of the glucocorticoid receptor and type $211 \beta$-hydroxysteroid dehydrogenase: potential molecular mechanisms underlying the programming of hypertension in utero. Endocrinology 142, 2841-2853.

25. Fernandez-Gonzalez R, Moreira P, Bilbao A, et al. (2004) Long-term effect of in vitro culture of mouse embryos with serum on mRNA expression of imprinting genes, development, and behavior. Proc Natl Acad Sci U S A 101, 5880-5885.

26. Kwong WY, Wild AE, Roberts P, et al. (2000) Maternal undernutrition during the preimplantation period of rat development causes blastocyst abnormalities and programming of postnatal hypertension. Development 127, 4195-4202.

27. Sinclair KD, Allegrucci C, Singh R, et al. (2007) DNA methylation, insulin resistance, and blood pressure in offspring determined by maternal periconceptional B vitamin and methionine status. Proc Natl Acad Sci U S A 104, 19351-19356.

28. Watkins AJ, Platt D, Papenbrock T, et al. (2007) Mouse embryo culture induces changes in postnatal phenotype including raised systolic blood pressure. Proc Natl Acad Sci U S A 104, 5449-5454.

29. Watkins AJ, Ursell E, Panton R, et al. (2008) Adaptive responses by mouse early embryos to maternal diet protect fetal growth but predispose to adult onset disease. Biol Reprod 78, 299-306.

30. Ceelen M, van Weissenbruch MM, Vermeiden JP, et al. (2008) Cardiometabolic differences in children born after in vitro fertilization: follow-up study. J Clin Endocrinol Metab 93, 1682-1688.

31. Rooke JA, McEvoy TG, Ashworth CJ, et al. (2007) Ovine fetal development is more sensitive to perturbation by the presence of serum in embryo culture before rather than after compaction. Theriogenology 67, 639-647.

32. Zander DL, Thompson JG \& Lane M (2006) Perturbations in mouse embryo development and viability caused by ammonium are more severe after exposure at the cleavage stages. Biol Reprod 74, 288-294.

33. Feil D, Lane M, Roberts CT, et al. (2006) Effect of culturing mouse embryos under different oxygen concentrations on subsequent fetal and placental development. J Physiol 572, 87-96.

34. Kwong WY, Miller DJ, Ursell E, et al. (2006) Imprinted gene expression in the rat embryo-fetal axis is altered in response to periconceptional maternal low protein diet. Reproduction 132, 265-277.

35. Kwong WY, Miller DJ, Wilkins AP, et al. (2007) Maternal low protein diet restricted to the preimplantation period induces a gender-specific change on hepatic gene expression in rat fetuses. Mol Reprod Dev 74, 48-56. 
36. Watkins AJ, Wilkins A, Cunningham C, et al. (2008) Low protein diet fed exclusively during mouse oocyte maturation leads to behavioural and cardiovascular abnormalities in offspring. J Physiol 586, 2231-2244.

37. Liu MY, Hattori Y, Fukao M, et al. (2001) Alterations in EDHF-mediated hyperpolarization and relaxation in mesenteric arteries of female rats in long-term deficiency of oestrogen and during oestrus cycle. Br J Pharmacol 132, 1035-1046.

38. Bertram JF (1995) Analyzing renal glomeruli with the new stereology. Int Rev Cytol 161, 111-172.

39. Vandesompele J, De Preter K, Pattyn F, et al. (2002) Accurate normalization of real-time quantitative RT-PCR data by geometric averaging of multiple internal control genes. Genome Biol 3, RESEARCH0034.

40. Watkins A, Wilkins A, Osmond C, et al. (2006) The influence of mouse Ped gene expression on postnatal development. J Physiol 571, 211-220.

41. Kwong WY, Osmond C \& Fleming TP (2004) Support for Barker hypothesis upheld in rat model of maternal undernutrition during the preimplantation period: application of integrated 'random effects' statistical model. Reprod Biomed Online 8, $574-576$

42. Lamireau D, Nuyt AM, Hou X, et al. (2002) Altered vascular function in fetal programming of hypertension. Stroke 33, 2992-2998.

43. Samuelsson AM, Matthews PA, Argenton M, et al. (2008) Dietinduced obesity in female mice leads to offspring hyperphagia, adiposity, hypertension, and insulin resistance: a novel murine model of developmental programming. Hypertension 51, 383-392.

44. Tang WJ \& Gilman AG (1992) Adenylyl cyclases. Cell 70, 869-872.

45. Longo M, Jain V, Vedernikov YP, et al. (2005) Fetal origins of adult vascular dysfunction in mice lacking endothelial nitric oxide synthase. Am J Physiol Regul Integr Comp Physiol 288, R1114-R1121.

46. Delpy E, Coste H \& Gouville AC (1996) Effects of cyclic GMP elevation on isoprenaline-induced increase in cyclic AMP and relaxation in rat aortic smooth muscle: role of phosphodiesterase. Br J Pharmacol 119, 471-478.

47. Kagota S, Tamashiro A, Yamaguchi Y, et al. (2001) Downregulation of vascular soluble guanylate cyclase induced by high salt intake in spontaneously hypertensive rats. $\mathrm{Br} \mathrm{J}$ Pharmacol 134, 737-744.

48. Kloss S, Bouloumie A \& Mulsch A (2000) Aging and chronic hypertension decrease expression of rat aortic soluble guanylyl cyclase. Hypertension 35, 43-47.

49. Ruetten H, Zabel U, Linz W, et al. (1999) Downregulation of soluble guanylyl cyclase in young and aging spontaneously hypertensive rats. Circ Res $\mathbf{8 5}, 534-541$.
50. Esther CR, Marino EM, Howard TE, et al. (1997) The critical role of tissue angiotensin-converting enzyme as revealed by gene targeting in mice. J Clin Invest 99, 2375-2385.

51. Klein JD, Le Quach D, Cole JM, et al. (2002) Impaired urine concentration and absence of tissue ACE: involvement of medullary transport proteins. Am J Physiol Renal Physiol 283, F517-F524.

52. Forrester T, McFarlane-Anderson N, Bennett FI, et al. (1997) The angiotensin converting enzyme and blood pressure in Jamaicans. Am J Hypertens 10, 519-524.

53. Nystrom F, Karlberg BE \& Ohman KP (1997) Serum angiotensin-converting enzyme activity correlates positively with plasma angiotensin II: a population-based study of ambulatory blood pressure and the renin-angiotensin system. J Hum Hypertens 11, 301-306.

54. Forhead AJ, Broughton Pipkin F \& Fowden AL (2000) Effect of cortisol on blood pressure and the renin-angiotensin system in fetal sheep during late gestation. $J$ Physiol 526, $167-176$.

55. Langley-Evans SC, Welham SJ \& Jackson AA (1999) Fetal exposure to a maternal low protein diet impairs nephrogenesis and promotes hypertension in the rat. Life Sci 64, 965-974.

56. Hoppe CC, Evans RG, Bertram JF, et al. (2007) Effects of dietary protein restriction on nephron number in the mouse. $A m J$ Physiol Regul Integr Comp Physiol 292, R1768-R1774.

57. Manalich R, Reyes L, Herrera M, et al. (2000) Relationship between weight at birth and the number and size of renal glomeruli in humans: a histomorphometric study. Kidney Int 58, 770-773.

58. Ortiz LA, Quan A, Zarzar F, et al. (2003) Prenatal dexamethasone programs hypertension and renal injury in the rat. Hypertension 41, 328-334.

59. Pladys P, Sennlaub F, Brault S, et al. (2005) Microvascular rarefaction and decreased angiogenesis in rats with fetal programming of hypertension associated with exposure to a low-protein diet in utero. Am J Physiol Regul Integr Comp Physiol 289, R1580-R1588.

60. Woods LL, Ingelfinger JR \& Rasch R (2005) Modest maternal protein restriction fails to program adult hypertension in female rats. Am J Physiol Regul Integr Comp Physiol 289, R1131-R1136.

61. Moritz KM \& Wintour EM (1999) Functional development of the meso- and metanephros. Pediatr Nephrol 13, 171-178.

62. Angiolini E, Fowden A, Coan P, et al. (2006) Regulation of placental efficiency for nutrient transport by imprinted genes. Placenta 27, S98-S102.

63. Smith FM, Garfield AS \& Ward A (2006) Regulation of growth and metabolism by imprinted genes. Cytogenet Genome Res 113, 279-291. 